\title{
Excitation spectrum of three dressed Bose-Einstein condensates
}

\author{
Zhong-Wen Ouyang and Le-Man Kuang* \\ Department of Physics, Hunan Normal University, Changsha 410081, China
}

\begin{abstract}
We study quantum dynamics of three dressed Bose-Einstein condensates in a high-Q cavity. The quasiparticle excitation spectrum of this system is found numerically. The stability of the quasiparticle excitation is analyzed. It is shown that there exist instabilities in the excitation spectrum.
\end{abstract}

Typeset using REVTEX 
Recently, multicomponent Bose-Einstein condensates (MBEC's) [1,2] have attracted much attention due to the recent experimental realizations of two and three component Bose-Einstein condensates (BEC's) [3,4]. MBEC's offer new degrees of freedom, which give rise to a rich set of new phenomena that do not exist in a single condensate. Especially, the interaction of BEC's with light leads to fascinating effects including the extreme slowing down of the speed of light [5] and matter-wave four-wave mixing [6]. Goldstein, Wright and Meystre (GWM) [0] proposed an approach to produce MBEC's, called dressed BEC's, inside a high- $Q$ multimode cavity, and investigated the quasiparticle excitation spectrum for the case of two dressed BEC's which dress one-photon. The purpose of this letter is to study the quasiparticle excitation spectrum for a system of three dressed BEC's which dress two photons. We will investigate quantum dynamics of the three dressed BEC's, and calculate numerically their elementary excitations.

Consider the GWM model of dressed BEC's [7], which consists of a condensate which interacts with two counter-propagating modes by a high-Q ring cavity with $n_{1}$ photons being in the mode 1 and $n_{2}$ photons being in the mode 2 . We consider a state composing $n=n_{1}+n_{2}$ photons and $N$ atoms which comprises the condensate confined by an external trapping potential $U(\mathbf{r})$. The state of the system can be written $\left|\Psi_{N, n}\right\rangle=$ $\sum_{n_{1}, n_{2}} \int d r_{1} \ldots \int d r_{N} f_{n_{1}, n_{2}}\left(\mathbf{r}_{1}, \ldots, r_{N}, t\right) \Psi^{\dagger}\left(\mathbf{r}_{1}\right) \ldots \Psi^{\dagger}\left(\mathbf{r}_{N}\right)$

$\times\left|0, n_{1}, n_{2}\right\rangle$, where $\Psi^{\dagger}\left(\mathbf{r}_{i}\right)$ are the atomic field operators, $f_{n_{1}, n_{2}}\left(\mathbf{r}_{1}, \ldots, r_{N}, t\right)$ is the manyparticle Schrödinger wave function for the condensate, which can be factorized as a product of Hartree wave functions $\phi_{n_{1}, n_{2}}$ representing states which the atoms occupy, $f_{n_{1}, n_{2}}\left(\mathbf{r}_{1}, \ldots, r_{N}, t\right)=\prod_{i=1}^{N} \phi_{n_{1}, n_{2}}\left(\mathbf{r}_{i}, t\right)$.

Let us consider such a trapping potential [8] $U(\mathbf{r})=m \omega_{\perp}^{2}\left(\mathbf{r}_{\perp}^{2}+\lambda^{2} z\right)^{2}$, where $r=\left(\mathbf{r}_{\perp}, z\right)$, $\mathbf{r}_{\perp}$ being the transverse position coordinate, $\omega_{\perp}$ the transverse angular frequency of the trap, and $\lambda=\omega_{z} / \omega_{L}$ is the ratio of the longitudinal to transverse frequencies. For the cigar structure condensate [9], it is reasonable to assume that $\lambda \ll 1$, and the transverse structure of the condensate is determined by the ground state solution of the transverse potential $v\left(\mathbf{r}_{\perp}\right)$. 
Then the Hartree wave function can be expressed as $\phi_{n_{1}, n_{2}}(\mathbf{r}, t)=v\left(\mathbf{r}_{\perp}\right) e^{-i \omega_{\perp} t} \psi_{n_{1}, n_{2}}(z, t)$. From the Hartree variational pronciple and after integrating over the transverse coordinate $r_{\perp}$, one obtains Gross-Pitaeviskii equations (GPE's)

$$
\begin{aligned}
i \dot{\psi}_{n_{1}, n_{2}}(\xi, \tau)= & H_{L} \psi_{n_{1}, n_{2}}(\xi, \tau) \\
& +\eta\left|\psi_{n_{1}, n_{2}}(\xi, \tau)\right|^{2} \psi_{n_{1}, n_{2}}(\xi, \tau) \\
& +g\left[\sqrt{n_{1}\left(n_{2}+1\right)} \psi_{n_{1}-1, n_{2}+1}(\xi, \tau)\right. \\
& \left.+\sqrt{\left(n_{1}+1\right) n_{2}} \psi_{n_{1}+1, n_{2}-1}(\xi, \tau)\right]
\end{aligned}
$$

where $H_{L}=-\partial^{2} / \partial \xi^{2}+\xi^{2} / 4$, with $\xi=z / a_{z}, a_{z}=\sqrt{\hbar / 2 m \omega_{z}}, \tau=\omega_{z} t, g$ and $\eta$ are the linear and nonlinear coupling coefficients, respectively.

The dressed BEC's are the eigenstates of Eq.(1), and are quantum superposition states of states with different photon numbers $\left(n_{1}, n_{2}\right)$. We consider the case of three dressed BEC's. In this case, only the states with $(2,0),(1,1)$ and $(0,2)$ are concerned. The coupled GPE's (1) reduce to

$$
\begin{aligned}
i \dot{\psi}_{2,0}(\xi, \tau) & =H_{L} \psi_{2,0}(\xi, \tau)_{+} \sqrt{2} g \psi_{1,1}(\xi, \tau) \\
& +\eta\left|\psi_{2,0}(\xi, \tau)\right|^{2} \psi_{2,0}(\xi, \tau), \\
i \dot{\psi}_{1,1}(\xi, \tau) & =H_{L} \psi_{1,1}(\xi, \tau)_{+} \sqrt{2} g\left[\psi_{2,0}(\xi, \tau)\right. \\
& \left.+\psi_{0,2}(\xi, \tau)\right]+\eta\left|\psi_{1,1}(\xi, \tau)\right|^{2} \psi_{1,1}(\xi, \tau), \\
i \dot{\psi}_{0,2}(\xi, \tau) & =H_{L} \psi_{0,2}(\xi, \tau)_{+} \sqrt{2} g \psi_{1,1}(\xi, \tau) \\
& +\eta\left|\psi_{0,2}(\xi, \tau)\right|^{2} \psi_{0,2}(\xi, \tau) .
\end{aligned}
$$

The exact analytic stationary solutions are generally not available for the coupling GPE's of the dressed BEC's. We here consider their solutions under the Thomas-Fermi approximation (TFA), in which the non-linear interaction term dominates over the kinetic-energy term [9]. Setting $\psi_{n_{1}, n_{2}}(\xi, \tau)=e^{-i \mu \tau} \theta_{n_{1}, n_{2}}(\xi)$ with $\mu$ being the chemical potential, under the TFA, coupling GPE's (2) reduce to

$$
\mu \theta_{2,0}(\xi)=\frac{1}{4} \xi^{2} \theta_{2,0}(\xi)+\sqrt{2} g \theta_{1,1}(\xi)+\eta\left|\theta_{2,0}(\xi)\right|^{2} \theta_{2,0}(\xi)
$$




$$
\begin{aligned}
\mu \theta_{0,2}(\xi)= & \frac{1}{4} \xi^{2} \theta_{0,2}(\xi)+\sqrt{2} g \theta_{1,1}(\xi)+\eta\left|\theta_{0,2}(\xi)\right|^{2} \theta_{0,2}(\xi) \\
\mu \theta_{1,1}(\xi)= & \frac{1}{4} \xi^{2} \theta_{1,1}(\xi)+\sqrt{2} g\left[\theta_{0,2}(\xi)+\theta_{2,0}(\xi)\right] \\
& +\eta\left|\theta_{1,1}(\xi)\right|^{2} \theta_{1,1}(\xi)
\end{aligned}
$$

For simplicity, we consider only solutions of two cases for $\theta_{2,0}(\xi)$ and $\theta_{0,2}(\xi)$ : (a) Out-ofphase solution with $\theta_{2,0}(\xi)=-\theta_{0,2}(\xi)$; (b) In-phase solution with $\theta_{2,0}(\xi)=\theta_{0,2}(\xi)$.

(a) Out-of-phase solution. In this case, $\theta_{2,0}(\xi)=-\theta_{0,2}(\xi)$, from Eqs.(3) we obtain the following dressed state nonzero solutions

$$
\theta_{2,0}(\xi)=\sqrt{\frac{4 \mu-\xi^{2}}{4 \eta^{2}}}, \quad \theta_{1,1}(\xi)=0, \quad 4 \mu \geq \xi^{2}
$$

Let $\xi_{m}$ be the boundary of the dressed condensates at which the TFA solutions vanish. From Eq.(4) it follows that the chemical potential $\mu=\frac{1}{4} \xi_{m}^{2}$. The value of $\xi_{m}$ is determined by the normalization condition of the condensate wave function $\sum_{n_{1}, n_{2}} \int_{-\xi_{m}}^{\xi_{m}} d \xi\left|\theta_{n_{1}, n_{2}}(\xi)\right|^{2}=1$, which leads to $\xi_{m}=[3 \eta / 2]^{1 / 3}$. So we get the following out-of-phase solution

$$
\left|\theta_{2,0}(\xi)\right|^{2}=\left|\theta_{0,2}(\xi)\right|^{2}=\frac{\xi_{m}^{2}-\xi^{2}}{4 \eta},\left|\theta_{1,1}(\xi)\right|^{2}=0 .
$$

It is interesting to note that the out-of-phase solution of the three dressed BEC's are the same as those of two dressed BEC's found in Ref. [7] due to the vanishness of $\theta_{1,1}(\xi)$. This reflects the fact that the three dressed BEC system can transit to a two dressed BEC system. Physically, this is reasonable, because a three dressed BEC system naturally becomes a two dressed BEC system when one of the three dressed condensates vanishes.

(b) In-phase solution. In the case, $\theta_{2,0}(\xi)=\theta_{0,2}(\xi)$, Eqs.(2) reduce to two equations:

$$
\begin{aligned}
\left(\mu-\frac{1}{4} \xi^{2}\right) \theta_{2,0}(\xi)= & \sqrt{2} g \theta_{1,1}(\xi)+\eta\left|\theta_{2,0}(\xi)\right|^{2} \theta_{2,0}(\xi) \\
\left(\mu-\frac{1}{4} \xi^{2}\right) \theta_{1,1}(\xi)= & 2 \sqrt{2} g \theta_{2,0}(\xi) \\
& +\eta\left|\theta_{1,1}(\xi)\right|^{2} \theta_{1,1}(\xi)
\end{aligned}
$$

which can be solved numerically. Assume $\xi_{m}$ to be the boundary of the dressed condensates, from Eqs.(6) we then obtain the chemical potential $\mu^{ \pm}= \pm 2 g+\xi_{m}^{2} / 4$. Here the value of $\xi_{m}$ 
depends on the normalization condition $\sum_{n_{1}, n_{2}} \int_{-\xi_{m}}^{\xi_{m}} d \xi\left|\theta_{n_{1}, n_{2}}(\xi)\right|^{2}=1$. For example, when $g=7.29$ and $\eta=80$, we numerically find that $\xi_{m}^{+}=4.584$ and $\xi_{m}^{-}=4.430$ coresponding to the chemical potential being $\mu^{+}$and $\mu^{-}$, respectively.

We now find the elementary excitations of the system by linearizing Eqs.(6) around the dressed state solutions: $\psi_{n_{1}, n_{2}}(\xi, \tau)=e^{-i \mu \tau}\left[\theta_{n_{1}, n_{2}}(\xi)+u_{n_{1}, n_{2}}(\xi) e^{-i \omega \tau}+v_{n_{1}, n_{2}}^{\star}(\xi) e^{i \omega \tau}\right]$, where $\left(n_{1}, n_{2}\right)=(2,0),(1,1)$ and $(0,2), u(\xi)$ and $v(\xi)$ represent small perturbations arround the dressed state with energies $\mu \pm \omega$. Substituting the expression of $\psi_{n_{1}, n_{2}}(\xi, \tau)$ into Eqs.(6), in first order, we get the system of six equations for the linearized perturbations $u(\xi)$ and $v(\xi):$

$$
\begin{aligned}
\omega u_{2,0}(\xi)= & {\left[H_{L}+2 \eta\left|\theta_{2,0}(\xi)\right|^{2}-\mu\right] u_{2,0}(\xi) } \\
& +\sqrt{2} g u_{1,1}(\xi)+\eta \theta_{2,0}(\xi)^{2} v_{2,0}(\xi), \\
\omega u_{1,1}(\xi)= & {\left[H_{L}+2 \eta\left|\theta_{1,1}(\xi)\right|^{2}-\mu\right] u_{1,1}(\xi) } \\
& +\sqrt{2} g\left[u_{2,0}(\xi)+u_{0,2}(\xi)\right]+\eta \theta_{1,1}(\xi)^{2} v_{1,1}(\xi), \\
\omega u_{0,2}(\xi)= & {\left[H_{L}+2 \eta\left|\theta_{0,2}(\xi)\right|^{2}-\mu\right] u_{0,2}(\xi) } \\
& +\sqrt{2} g u_{1,1}(\xi)+\eta \theta_{0,2}(\xi)^{2} v_{0,2}(\xi), \\
-\omega v_{2,0}(\xi)= & {\left[H_{L}+2 \eta\left|\theta_{2,0}(\xi)\right|^{2}-\mu\right] v_{2,0}(\xi) } \\
& +\sqrt{2} g v_{1,1}(\xi)+\eta \theta_{2,0}(\xi)^{2} u_{2,0}(\xi), \\
-\omega v_{1,1}(\xi)= & {\left[H_{L}+2 \eta\left|\theta_{1,1}(\xi)\right|^{2}-\mu\right] v_{1,1}(\xi) } \\
& +\sqrt{2} g\left[v_{2,0}(\xi)+v_{0,2}(\xi)\right]+\eta \theta_{1,1}(\xi)^{2} u_{1,1}(\xi), \\
-\omega v_{0,2}(\xi)= & {\left[H_{L}+2 \eta\left|\theta_{0,2}(\xi)\right|^{2}-\mu\right] v_{0,2}(\xi) } \\
& +\sqrt{2} g v_{1,1}(\xi)+\eta \theta_{0,2}(\xi)^{2} u_{0,2}(\xi) .
\end{aligned}
$$

The normal modes of the above coupled equations are identical to the elemantary excitations determined via Bogoliubov method. In order to obtain the elementary excitations, we expand them in terms of eigenfunctions of the trapping potential $q_{\nu}(\xi)$ as

$$
u_{n_{1}, n_{2}}(\xi)=\sum_{\nu} b_{n_{1}, n_{2}}^{\nu} q_{\nu}(\xi)
$$




$$
v_{n_{1}, n_{2}}(\xi)=\sum_{\nu} c_{n_{1}, n_{2}}^{\nu} q_{\nu}(\xi)
$$

where $q_{\nu}(\xi)$ satisfies $H_{L} q_{\nu}(\xi)=\left(\nu+\frac{1}{2}\right) q_{\nu}(\xi)$.

It is difficult to get an exact solution for the normal modes of Eqs.(7). In what follows, we give numerical solution of them by using the TFA. For the out-of-phase solution (4), we note that $\left|\theta_{2,0}(\xi)\right|^{2}=\left|\theta_{0,2}(\xi)\right|^{2} \simeq\left|\theta_{2,0}(\xi=0)\right|^{2} \approx \xi_{m}^{2} / 4 \eta$ for normal modes localized close to the center of the trapping potential due to $\xi_{m} \gg 1$.

Substitution of Eqs.(8) into Eqs.(7) gives a system of linear equations for the coefficients $b_{n_{1}, n_{2}}^{\nu}$ and $c_{n_{1}, n_{2}}^{\nu}$. The nonzero solution condition of the system of linear equations gives rise to the excitation spectrum of the elementary excitations. In the Fig. 1(a) we have plotted the excitation spectrum in terms of the index $\nu$ of the linear oscillator mode for the out-ofphase solution when $\eta=80, g=7.29$ and $\eta_{m}=4.9324$. Fig. $1(\mathrm{~b})$ is the enlargement of the part of $-10<\omega^{2}<20$ for the curve $c$. From Fig.1(a) we see that in the region of $0 \leq \nu \leq 2$, there exists one type of excitation spectrum, which is stable due to $\omega^{2}>0$. In the region of $\nu>2$ we can observe three types of excitation spectrum. Two of them indicated by curve $a$ and $b$ are stable excitations. The third one indicated by curve $c$ is instable in the region of $14<\nu<18$ since $\omega^{2}$ is negative. This is explicitly indicated in Fig. 1(b) through the enlargement of the curve $c$.

Similarly, we can numerically express the excitation spectrum for the in-phase solution in terms of the index $\nu$ of the linear oscillator mode. In Fig. 2, we give rise to the excitation spectrum for the in-phase solution when $\eta=80, g=7.29$ and $\eta_{m}=4.932$, where we have set that $\left|\theta_{2,0}(\xi)\right|^{2}=\left|\theta_{0,2}(\xi)\right|^{2} \simeq\left|\theta_{2,0}(\xi=0)\right|^{2} \approx 0.0336,\left|\theta_{1,1}(\xi)\right|^{2} \simeq\left|\theta_{1,1}(\xi=0)\right|^{2} \approx 0.0925$ for the branch $\mu^{+}$, and $\left|\theta_{2,0}(\xi)\right|^{2}=\left|\theta_{0,2}(\xi)\right|^{2} \approx 0.04925,\left|\theta_{1,1}(\xi)\right|^{2}=\left|\theta_{1,1}(0)\right|^{2} \approx 0.0775$ for the branch $\mu^{-}$, which lead to $\xi_{m}^{+}=4.584$ and $\xi_{m}^{-}=4.431$, respectively. From Fig. 2(a), we see that there exist different excitation spectrum for three different regions of $\nu$. In the first region $0<\nu<8$, we can find three kinds of excitation spectrum, and all of them are stable. In the second region $8<\nu<13$, there is only one kind of excitation spectrum which is unstable (i.e., $\omega^{2}<0$ ). In the third region $\nu>13$, there exist three kinds of excitation 
spectrum. One of them is stable, and other two are unstable. These unstable regimes are explicitly indicated in Fig. 2(b), which is the enlargement of the curves in the vicinity of the point A in Fig. 2(a). From Fig. 2(c), we see that $\omega^{2}$ has two positive real number solutions and one pure imaginary solution which is not indicated in the figure. these means that there does not exist solutions of $\omega^{2}<0$. Therefore, the excitation spectrum of this case is always stable.

This work is supported in part by the National Climbing Program of China and NSF of China, the Excellence Young-Teacher Foundation of the Educational Department of China, and ECF and STF of Hunan Province. The authors thank Dr.Changsong Zhou for his help in preparing figures of the paper.

* Corresponding author. 


\section{REFERENCES}

[1] T.-L. Ho and V. B. Shenoy, Phys. Rev. Lett. 77 (1996) 3276; B. D. Esry, C. H. Greene, et al. , ibid. 78 (1997) 3594; E. V. Goldstein and P. Meystre, Phys. Rev. A55 (1997) 2935; T. Busch, J. I. Cirac, et al., Phys. Rev. A56 (1997) 2978.

[2] C. K. Law, H. Pu, et al., Phys. Rev. Lett. 79 (1997) 3105; H. Pu and N. P. Biegelow, ibid. 80 (1998) 1130; L. M. Kuang and Z. Y. Zeng, Chin. Phys. Lett. 15 (1998) 703; L. M. Kuang, et al., Phys. Rev. A61 (2000) 013608; ibid. A61 (2000) 023604.

[3] C. J. Myatt, E. A. Burt, et al., Phys. Rev. Lett. 78 (1997) 586; D. M. Stamper-Kurn, M. R. Andrews, et al., ibid. 80 (1998) 2027.

[4] M. H. Anderson, et al., Science 269 (1995) 198; K. B. Davis, et al., Phys. Rev. Lett. 75 (1995) 3969. C. C. Bradley, et al., Phys. Rev. Lett. 75 (1995) 1687.

[5] L. V. Hau, et al., Nature 398 (1999) 218.

[6] L. Deng, et al., Nature 397 (1999) 594.

[7] E. V. Goldstein, Ewan M. Wright, and P. Meystre, Phys. Rev. A57 (1998) 1223.

[8] M. Edwards and K. Burnett, Phys. Rev. A51 (1995) 1382; G. Baym and C. J. Pethick, Phys. Rev. Lett. 76 (1996) 6.

[9] M.-O. Mewes, M. R. Andrews, et al., Phys. Rev. Lett. 77 (1996) 416; M. Edwards, and K. Burnett, Phys. Rev. A51 (1995) 1382; P. A. Ruprecht, M. J. Holland, et al., ibid. A51 (1995) 4704 .

\section{Figure Captions}

FIG.1. (a) Excitation spectrum $\omega^{2}$ for the out-of-phase solution for $\eta=80, g=7.29$, and $\eta_{m}=4.932$. (b) The enlargement of the part of $-10<\omega^{2}<20$ for the curve $c$.

FIG.2. (a) Excitation spectrum $\omega^{2}$ for the in-phase solution with $\xi_{m}^{+}=4.584$. (b) The enlargement of the curves in the vicinity of the point A in Fig.1(a). (c) Excitation spectrum 
$\omega^{2}$ for the in-phase solution with $\xi_{m}^{-}=4.431$. Here we set $\eta=80, g=7.29$. 

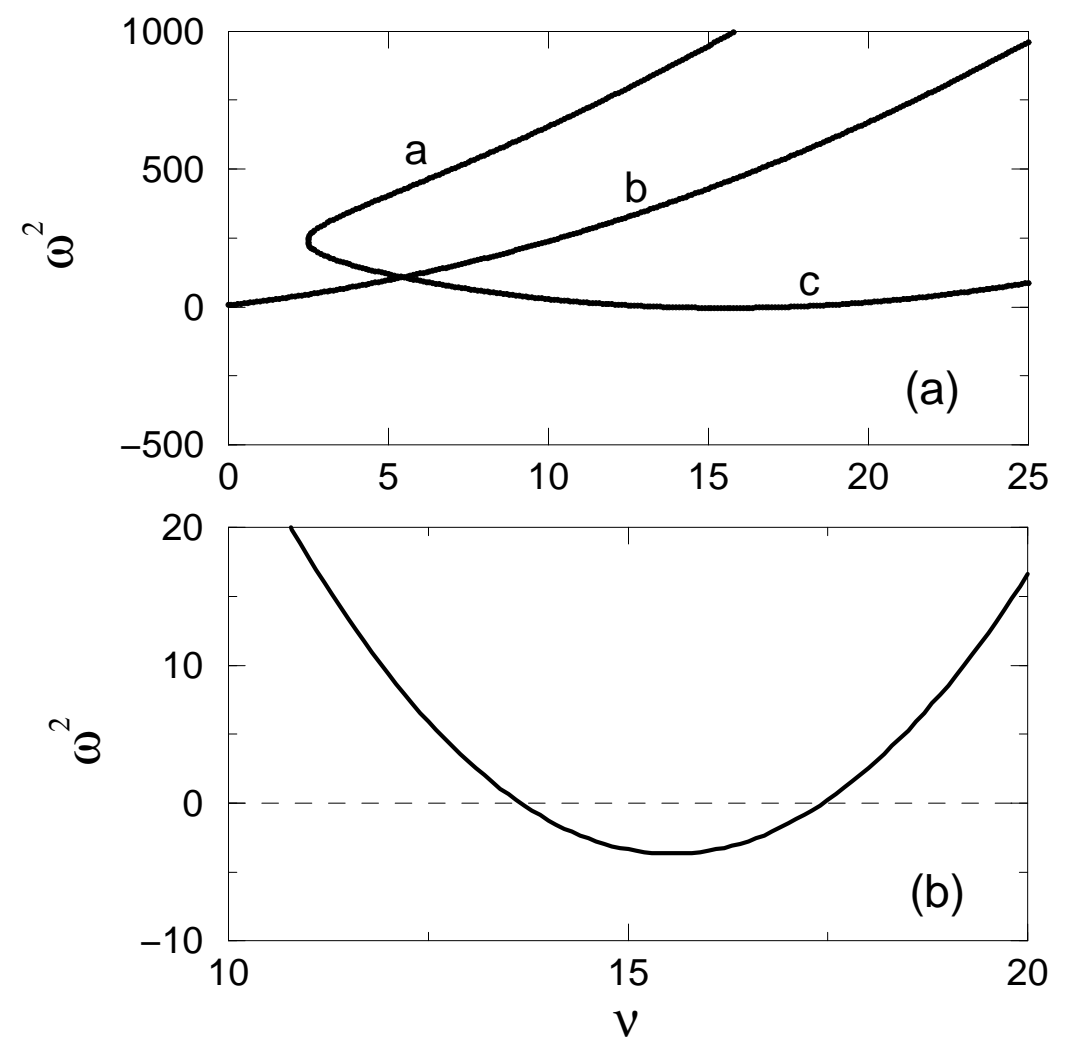

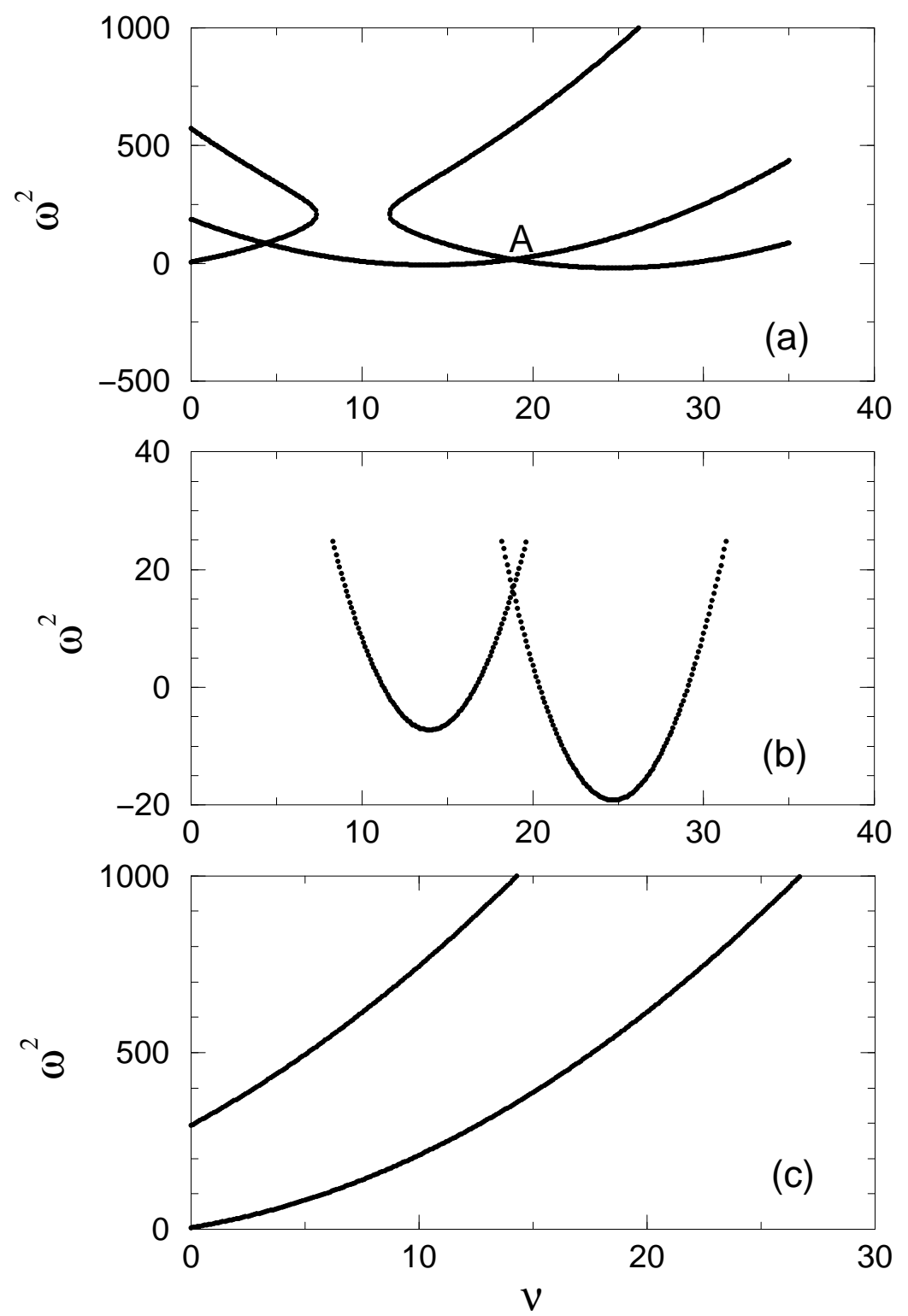